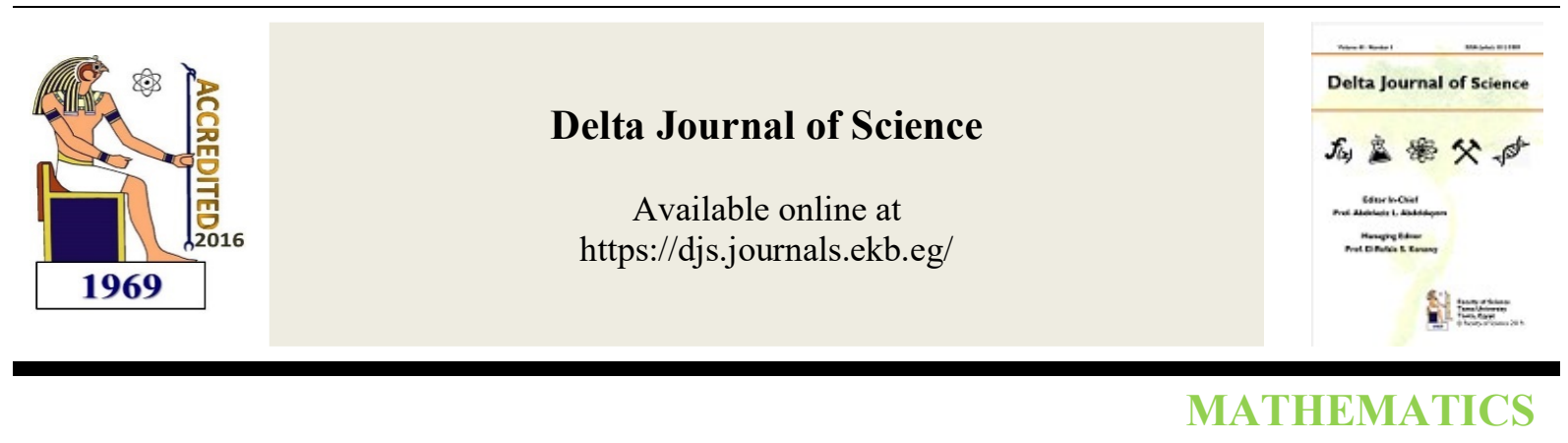

\title{
A new Method to Estimate the Parameters of Quadratic Regression
}

\author{
M.A. Kassem ${ }^{a, *}$, A.M. Salem ${ }^{\text {b,* }}$ and N.G. Ragab ${ }^{\mathrm{c}, *}$ \\ Department of Mathematics, Faculty of Science, Tanta University, Tanta, Egypt \\ KEY WORDS \\ Quadratic \\ Regression, \\ Kuhn-Tucker \\ conditions, Auto- \\ correlation. \\ ABSTRACT \\ In this study, a new method to estimate the parameters for a quadratic \\ regression model is introduced by using Kuhn-Tucker conditions. \\ Kuhn-Tucker conditions provide the minimizing error of the estimated \\ parameters for a quadratic regression. This method can be used for any \\ data set of a quadratic regression, and we discuss the test for correct \\ specification of disturbances mainly because of their ability to detect \\ the irregularities in the regressor specification.
}

\section{Introduction}

A quadratic regression refers to linear regression with two or more predictors $\left(x_{1}, x_{2}, \ldots, x_{n}\right)$. When multiple predictors are used, the regression line cannot be visualized in two-dimensional space. However, the line can be computed simply by expanding the equation for single-predictor linear regression to include the parameters for each of the predictors.

Although linearity is still the primary model in applications, there has been an increasing in examples of data that are nonlinear, and in particular, where a quadratic fit may be more appropriate. The method of Theil [9] can readily be modified for the quadratic case.

Necessary and sufficient conditions for noninferiority due to Kuhn and Tucker are analogous to the classic Kuhn-Tucker conditions for optimality of a scalar optimization problem. The Kuhn-Tucker conditions for noninferiority (KTCN) will be 
defined in the same spirit as in Cohon and Marks [3] and Cohon [2].

A quadratic regression models play an important role in many fields. The object of this paper is to estimate the parameters for a quadratic regression model by using KuhnTucker conditions. Kuhn-Tucker conditions provide the minimize error of the estimated parameters for a quadratic regression.

The Durbin-Watson statistic is a statistical test used to detect the presence of autocorrelation in the residuals (prediction errors) from a regression analysis [9, 10]. Durbin and Watson $(1950,1951)$ applied this statistic to the residuals from the regression line, and developed bounds tests for the null hypothesis that the errors are serially uncorrelated against the alternative that they follow a first order autoregressive process.

A quadratic regression models which studied by using Kuhn-Tucker conditions can take the following form:

$$
y_{i}=\beta_{1} x_{i}+\beta_{2} x_{i}^{2}+\varepsilon_{i} \quad, i=1,2, \ldots, n
$$

where $y_{i}, x_{i}$ and $\left(\beta_{1}, \beta_{2}\right)$ are vectors of endogenous variables, exogenous variables, and regression parameters to be estimated, respectively, and $\varepsilon_{i}$ is a random error, assumed to be normally distributed, independently of the errors for other observations, with expectation 0 and variance $\sigma^{2}: \varepsilon_{i} \approx N\left(0, \sigma^{2}\right)$.

The purpose of this article is to develop a new procedure that can always produce regression curve estimators for the quadratic model (1.1) by using the Kuhn-Tucker conditions.
Equation (1.1) can be written in the following form:

$$
\begin{aligned}
& \min Q=\sum_{i=1}^{n} \varepsilon_{i}^{2} \quad, i=1, \ldots, n \\
& \text { s.t. } \\
& \sum_{i=1}^{n}\left[y_{i}-\left(\beta_{1} x_{i}+\beta_{2} x_{i}^{2}\right]^{2} \leq Q,\right. \\
& \quad x_{i} \geq 0
\end{aligned}
$$

Definition1 [1]:

Let $f_{i}: R^{n} \rightarrow R, g_{i}: R^{n} \rightarrow R$ and $S=\left\{x \in R^{n}: g_{i} \leq 0\right\}$, a feasible solution $x \in S$ is said to be satisfy KTCN for vector optimization problem if:

1. all $f_{i}$ and $g_{i}$ are differentiable and $S=\phi$; and

2. there exists $u_{i} \geq 0, i=1, \ldots, n$, with strict inequality holding for at least one $\square_{k}$ and $v_{i} \geq 0, i=1, \ldots, n$, such that

$$
g_{i}(x) \leq 0, \quad v_{i} g_{i}(x)=0 \quad(i=1, \ldots, n),
$$

and

$$
\sum_{i=1}^{n} u_{i} \nabla f_{i}(x)+\sum_{i=1}^{n} v_{i} \nabla g_{i}(x)=0 .
$$

The Kukn-Tucker conditions for this problem take the form (see $[6,7])$ :

$u_{i}\left[-2 \sum_{i=1}^{n}\left[y_{i}-\left(\hat{\beta}_{1} x_{i}+\hat{\beta}_{2} x_{i}^{2}\right)\right]\left(\hat{\beta}_{1}+2 \hat{\beta}_{2} x_{i}\right)\right]-\sum_{i=1}^{n} v_{i} x_{i}=0$,

$\sum_{i=1}^{n} u_{i}=1$

$\sum_{i=1}^{n}\left[y_{i}-\left(\hat{\beta}_{1} x_{i}+\hat{\beta}_{2} x_{i}^{2}\right)\right]^{2} \leq 0$,

$-\sum_{i=1}^{n} v_{i} x_{i}=0$

\section{Problem Formulation}


$u_{i}\left[\sum_{i=1}^{n}\left[y_{i}-\left(\hat{\beta}_{1} x_{i}+\hat{\beta}_{2} x_{i}^{2}\right)\right]^{2}\right]=0$,

$u_{i} \geq 0, \quad i=1, \ldots, n$

$v_{i} \geq 0$

The determination of $\hat{\beta}_{1}, \hat{\beta}_{2}$ is depending on the obtained values of $u_{i}$ which can be determined as follow:

If $u_{i}>0, v_{i}=0$, we have:

$$
\hat{\beta}_{1}=\frac{\bar{y}}{\bar{x}}-\frac{\hat{\beta}_{2}}{n \bar{x}} \sum_{i=1}^{n} x_{i}^{2}, \quad \hat{\beta}_{2}=\frac{\sum_{i=1}^{n} x_{i} y_{i}-\frac{\bar{y}}{\bar{x}} \sum_{i=1}^{n} x_{i}^{2}}{\sum_{i=1}^{n} x_{i}^{3}-\frac{\left(\sum_{i=1}^{n} x_{i}^{2}\right)^{2}}{n \bar{x}}} .
$$

\section{Stability set of this problem}

Given certain $\left(\hat{\beta}_{1}, \hat{\beta}_{2}\right)$ with corresponding optimal solution $e_{i}$; then the stability set of the first kind of problem (2.1) corresponding to this optimal solution, denoted by $S\left(e_{i}\right)$, is defined by $S\left(e_{i}\right)=\left\{\left(\hat{\beta}_{1}, \hat{\beta}_{2}\right) \in R \mid \mathrm{e}_{i} \quad\right.$ is an optimal solution of $(2.1)\}$ (see $[6,7])$.

For a certain $\left(\hat{\beta}_{1}, \hat{\beta}_{2}\right)$ with a corresponding to optimal solution $e_{i}$; we have from the stability of (2.1) there exist $\left(\hat{\beta}_{1}, \hat{\beta}_{2}\right) \in R, u_{i} \geq 0, i=1, \ldots, n$, such that the Kuhn-Tucker conditions of problem (2.1) takes the form (2.2)-(2.9), the determine of the stability set of the first kind $S\left(e_{i}\right)$ depends only on whether any of the variables $u_{i}, i=1, \ldots, n$ and any of the variables $v, w$ which solves the Equations (2.2), (2.3), (2.8), (2.9) are positive or zero.
Let $u_{i}=0, i \in I \subset\{1, \ldots, n\}, u_{i}>0, \quad i \notin I \quad$ solves (2.1), (2.2) and (2.7), then in order to satisfy the other Kuhn-Tucker conditions (2.3) and (2.6), we must have

$$
\begin{aligned}
& e_{i}=y_{i}-\left(\hat{\beta}_{1} x_{i}+\hat{\beta}_{2} x_{i}^{2}\right), i \notin I ; \\
& e_{i} \geq y_{i}-\left(\hat{\beta}_{1} x_{i}+\hat{\beta}_{2} x_{i}^{2}\right), i \in I .
\end{aligned}
$$

Let

$$
D=\left\{I \mid \mathrm{u}_{i}=0, i \in I ; \mathrm{u}_{i}>0, \quad i \notin I\right. \text { solve (2.1), }
$$

(2.2), (2.7) and (2.8)\}

and

$$
\begin{aligned}
S_{I}\left(e_{i}\right)= & \left\{\left(\hat{\beta}_{1}, \hat{\beta}_{2}\right) \in R \mid e_{i}=y_{i}-\left(\hat{\beta}_{1} x_{i}+\hat{\beta}_{2} x_{i}^{2}\right), i \notin I ;\right. \\
& \left.e_{i} \geq y_{i}-\left(\hat{\beta}_{1} x_{i}+\hat{\beta}_{2} x_{i}^{2}\right), i \in I\right\} .
\end{aligned}
$$

Then, it is clear that

$$
S\left(e_{i}\right)=\underset{I \in D}{ } S_{I}\left(e_{i}\right)
$$

\section{Testing Disturbances}

In this study we examine whether the disturbances in the regression model (2.1) are well behaved or not. As known, this can also be viewed as a test for the higher moments of the dependent variable conform to the assumptions of the model.

Testing for autocorrelation may be the most intensely researched statistical problems in all of econometrics. Despite heavy competitions, the most common procedure is still the DurbinWatson test. It is based on the assumption that the $e_{i}$ in (2.1) follows a stationary autoregressive process.

The null hypothesis of no serial correlation is therefore equivalent to $H_{0}: \rho=0$. The statistic test in the multivariate case as the Durbin-Watson test is 


$$
D=\frac{\sum_{i=2}^{n}\left(e_{i}-e_{i-1}\right)^{2}}{\sum_{i=1}^{n} e_{i}^{2}}
$$

where $e_{i}=y_{i}-\hat{y}_{i}$ is residuals, $y_{i}$ and Jare, respectively, the observed and predicted values of the response variable for individual $i$. A major problem with the Durbin-Watson test used to be that the rejection region depends not only on the significance level $\alpha$ of the test, but also on the regression vector $X$. Durbin et. al. [5] gave the familiar bounds $D_{U}$ and $x$ for which depends only on $\alpha, n$ such that (when testing against positive serial correlation)
If $D<D_{L}$
reject $H_{0}: \rho=0$
If $D>D_{U}$
do not reject $H_{0}: \rho=0$
If $D_{L}<D<D_{U}$
test is inconclusive.

\section{Example}

In this example we examined whether the disturbances in the regression median model (2.1) are well behaved or not. According to Durbin-Watson test, therefore the null hypothesis of no serial correlation $\left(H_{0}: \rho=0\right)$, against $\left(H_{1}: \rho>0\right)$.

The data set of $X$ and $Y$ is given in table 1

Table 1:

\begin{tabular}{|c|c|c|c|c|c|c|}
\hline$i$ & $x_{i}$ & $y_{i}$ & $\hat{y}_{i}(\mathrm{KT})=4.235^{*} x_{i}+0.25^{*} x_{i}^{2}$ & $e_{i}=y_{i}-\hat{y}_{i}$ & $e_{i}^{2}$ & $e$ \\
\hline 1 & 3.4 & 35 & 17.289 & 17.711 & 313.6795 & \\
\hline 2 & 5.7 & 40 & 32.262 & 7.738 & 59.87664 & - \\
\hline 3 & 7.5 & 42.5 & 45.825 & -3.325 & 11.05563 & - \\
\hline 4 & 8.8 & 44 & 56.628 & -12.628 & 159.4664 & - \\
\hline 5 & 11.1 & 60.5 & 77.811 & -17.311 & 299.6707 & - \\
\hline 6 & 12.5 & 80 & 92 & -12 & 144 & $\vdots$ \\
\hline 7 & 14.2 & 120 & 110.547 & 9.453 & 89.35921 & 2 \\
\hline 8 & 15.2 & 125 & 122.132 & 2.868 & 8.225424 & - \\
\hline 9 & 15.8 & 132 & 129.323 & 2.677 & 7.166329 & - \\
\hline 10 & 17.9 & 140 & 155.909 & -15.909 & 253.0963 & - \\
\hline 11 & 18 & 145 & 157.23 & -12.23 & 149.5729 & $\vdots$ \\
\hline 12 & 18.2 & 160 & 159.887 & 0.113 & 0.012769 & 1 \\
\hline 12 & 10 & 174 & 170715 & 2285 & 1070172 & $:$
\end{tabular}

By using equation (4.1) the statistic test is $D(\mathrm{KT})=0.77$, at significances level $\alpha=0.05$, then table 2 gives the critical values corresponding to $x$ and one regressor as $D_{L}=1.08$ and $\mathrm{x}$

Since $x$, then we reject $H_{0}: \rho=0$ and conclude that the errors are positively autocorrelated.

Table 2: critical values of the DurbinWatson statistic

\begin{tabular}{|c|c|c|c|c|c|c|c|c|c|}
\hline \multirow{2}{*}{$\begin{array}{l}\text { Sample } \\
\text { size (n) }\end{array}$} & \multirow{2}{*}{$\begin{array}{c}\text { (Significance } \\
\text { Level- } a \text { ) }\end{array}$} & \multicolumn{2}{|c|}{$k=1$} & \multicolumn{2}{|c|}{$k=2$} & \multicolumn{2}{|c|}{$k=3$} & \multicolumn{2}{|c|}{$k=4$} \\
\hline & & $\mathrm{DL}$ & $\mathrm{DU}$ & $\mathrm{DL}$ & $\mathrm{DU}$ & $\mathrm{DL}$ & $\mathrm{DU}$ & $\overline{\mathrm{DL}}$ & \\
\hline \multirow{3}{*}{15} & 0.01 & 0.81 & 1.07 & 0.7 & 1.25 & 0.59 & 1.46 & 0.49 & \\
\hline & 0.025 & 0.95 & 1.23 & 0.83 & 1.4 & 0.71 & 1.61 & 0.59 & \\
\hline & 0.05 & 1.08 & 1.36 & 0.95 & 1.54 & 0.82 & 1.75 & 0.69 & \\
\hline \multirow{3}{*}{20} & 0.01 & 0.95 & 1.15 & 0.86 & 1.27 & 0.77 & 1.41 & 0.63 & \\
\hline & 0.025 & 1.08 & 1.28 & 0.99 & 1.41 & 0.89 & 1.55 & 0.79 & \\
\hline & 0.05 & 1.2 & 1.41 & 1.1 & 1.54 & 1 & 1.68 & 0.9 & \\
\hline \multirow{3}{*}{25} & 0.01 & 1.05 & 1.21 & 0.98 & 1.3 & 0.9 & 1.41 & 0.83 & \\
\hline & 0.025 & 1.13 & 1.34 & 1.1 & 1.43 & 1.02 & 1.54 & 0.94 & \\
\hline & 0.05 & 1.29 & 1.45 & 1.21 & 1.55 & 1.12 & 1.66 & 1.04 & \\
\hline \multirow{3}{*}{30} & 0.01 & 1.13 & 1.26 & 1.07 & 1.34 & 1.01 & 1.42 & 0.94 & \\
\hline & 0.025 & 1.25 & 1.38 & 1.18 & 1.46 & 1.12 & 1.54 & 1.05 & \\
\hline & 0.05 & 1.35 & 1.49 & 1.28 & 1.57 & 1.21 & 1.65 & 1.14 & \\
\hline \multirow{3}{*}{40} & 0.01 & 1.25 & 1.34 & 1.2 & 1.4 & 1.15 & 1.46 & 1.1 & \\
\hline & 0.025 & 1.35 & 1.45 & 1.3 & 1.51 & 1.25 & 1.57 & 1.2 & \\
\hline & 0.05 & 1.44 & 1.54 & 1.39 & 1.6 & 1.34 & 1.66 & 1.29 & \\
\hline \multirow{3}{*}{50} & 0.01 & 1.32 & 1.4 & 1.28 & 1.45 & 1.24 & 1.49 & 1.2 & \\
\hline & 0.025 & 1.42 & 1.5 & 1.38 & 1.54 & 1.34 & 1.59 & 1.3 & \\
\hline & 0.05 & 1.5 & 1.59 & 1.46 & 1.63 & 1.42 & 1.67 & 1.38 & \\
\hline \multirow{3}{*}{60} & 0.01 & 1.38 & 1.45 & 1.35 & 1.48 & 1.32 & 1.52 & 1.28 & \\
\hline & 0.025 & 1.47 & 1.54 & 1.44 & 1.57 & 1.4 & 1.61 & 1.37 & \\
\hline & 0.05 & 1.55 & 1.62 & 1.51 & 1.65 & 1.48 & 1.69 & 1.44 & \\
\hline
\end{tabular}




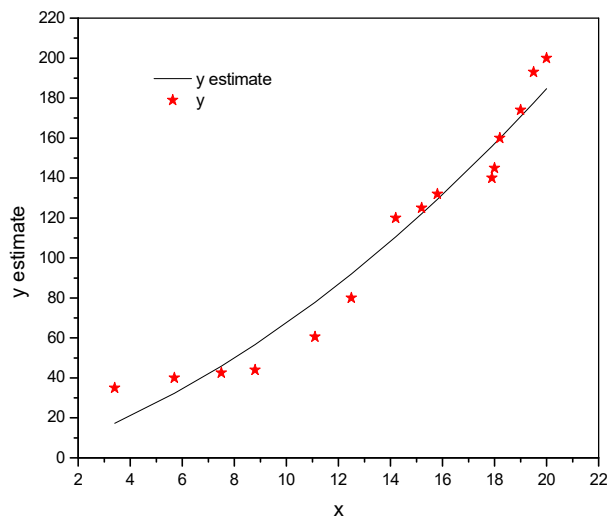

Fig. (1) estimated quadratic regression by Kuhn-Tucker conditions

\section{Comparison of Kuhn-Tucker Estimation and Least Squares Estimation on Quadratic Regression}

The data in table 1 has been studied by using least square method.

By using SPSS program for this example, we get the following analysis data:

$$
\hat{\beta}_{1}=3.062, \quad \hat{\beta}_{2}=0.320 .
$$

Then the quadratic regression equation estimated by least square (LS) method given by:

$$
\hat{y}_{i}(\mathrm{LS})=(3.062) x_{i}+(0.320) x_{i}^{2} \quad, i=1, \ldots, 15
$$

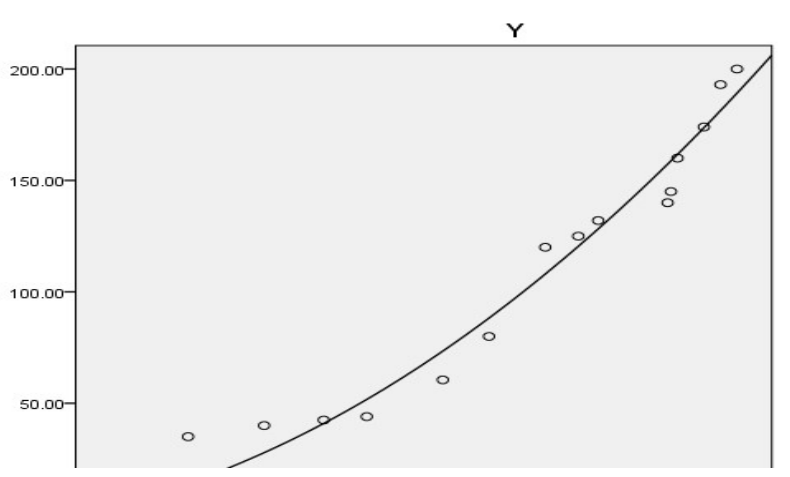

Fig. (2): estimated quadratic regression by least square method
The estimated quadratic regression is illustrated in Fig. (3) as a comparison between KuhnTucker (KT) conditions and least square (LS) method

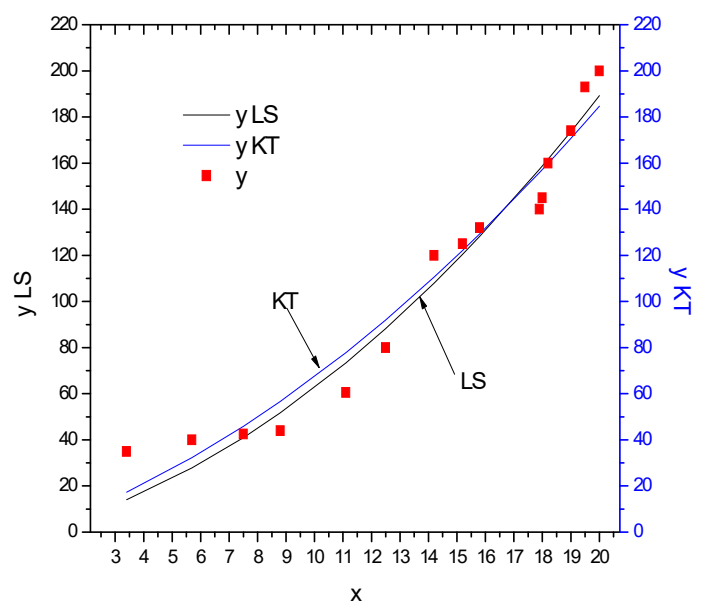

Fig. (3): estimated quadratic regression by Kuhn-Tucker (KT) conditions and least square (LS) method

Residual values can be calculated as shown in the following table (3).

Table (3):

\begin{tabular}{|c|c|c|c|c|c|}
\hline$i$ & $y_{i}$ & $\hat{y}_{i}(\mathrm{LS})$ & $e_{i}=y_{i}-\hat{y}_{i}$ & $e_{i}{ }^{2}$ & $e_{i}-e_{i-1}$ \\
\hline 1 & 35 & 14.11 & 20.89 & 436.3921 & \\
\hline 2 & 40 & 27.8502 & 12.1498 & 147.6176 & -8.7402 \\
\hline 3 & 42.5 & 40.965 & 1.535 & 2.356225 & -10.6148 \\
\hline 4 & 44 & 51.7264 & -7.7264 & 59.69726 & -9.2614 \\
\hline 5 & 60.5 & 73.4154 & -12.9154 & 166.8076 & -5.189 \\
\hline 6 & 80 & 88.275 & -8.275 & 68.47563 & 4.6404 \\
\hline 7 & 120 & 108.0052 & 11.9948 & 143.8752 & 20.2698 \\
\hline 8 & 125 & 120.4752 & 4.5248 & 20.47382 & -7.47 \\
\hline 9 & 132 & 128.2644 & 3.7356 & 13.95471 & -0.7892 \\
\hline 10 & 140 & 157.341 & -17.341 & 300.7103 & -21.0766 \\
\hline 11 & 145 & 158.796 & -13.796 & 190.3296 & 3.545 \\
\hline 12 & 160 & 161.7252 & -1.7252 & 2.976315 & 12.0708 \\
\hline 13 & 174 & 173.698 & 0.302 & 0.091204 & 2.0272
\end{tabular}

By using equation (4.1) the statistic test is $D(\mathrm{LS})=0.85$, at significances level $\mathrm{x}$, 
table 2 gives the critical values corresponding to $n=15$ and one regressor as $x \quad$ and $D_{U}=1.36$.

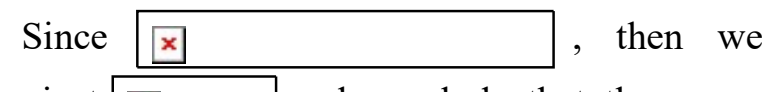
reject $x \quad$ and conclude that the errors are positively autocorrelated.

The result shows that, the statistic DurbinWatson test value in Kuhn-Tucker estimation

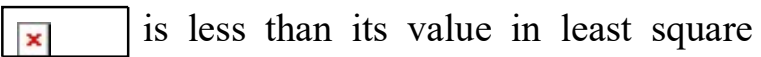
estimation $\mathrm{x}$.

\section{Conclusion}

In this paper, we introduce a new method to estimate the parameter for a quadratic regression model by using Kuhn-Tucker conditions. According to the Durbin-Watson test we show that there is positively autocorrelation between the errors for the regression curve, this means that our estimators are a suitable estimator in the case of fitting data.

\section{References}

1- V. Chankong and Yacov Y. Haimes, Multiobjective Decision Making: Theory and Methodology, New York, 1983.

2- J. L. Cohon, Multiobjective Programming and Planning, Academic, New York, 1978.

3- J. L. Cohon and D. H. Marks, A review and evaluation of multiobjective programming techniques, Water Resources Research 11, pp. 208-220,1975.

4- J. Durbin and G. S. Watson, "Testing for Serial Correlation in Least Squares Regression, I". Biometrika 37 (3-4): 409428, 1950.

5- J. Durbin and G. S. Watson, "Testing for Serial Correlation in Least Squares Regression, II". Biometrika 38 (1-2): 159179, 1951.

6- M. Kassem, Interactive stability of multiobjective nonlinear programming problems with fuzzy parameters in the constraints, Fuzzy Sets and Systems 73: 235-243, 1995.

7- M. Kassem, Interactive stability cuttingplane algorithm for multiobjective nonlinear programming problems, Applied Mathematics and Computation 192: 446456, 2007.

8- J. V. Neumann, "Distribution of the ratio of the mean square successive difference to the variance". Annals of Mathematical Statistics 12 (4): 367-395, 1941.

9- A. M. Salem, Testing for serial correlation in multivariate least absolute deviations regression. International formation institute10 (6): 777-780, 2007.

10- A. M. Salem, An efficient for regression median. International Journal of pure and applied mathematics 37 (4): 543-555, 2007.

11- H. Theil, A rank-invariant method of linear and polynomial regression analysis. I, II, III. Proceedings Nederlandse Akademie van Wetenschappen 53: 386-392, 521-525, 1397-1412, 1950. 